\title{
Distribution of Gaps and Adhesive Interaction Between Contacting Rough Surfaces
}

\author{
Joseph M. Monti ${ }^{1,2}$ (D) Antoine Sanner ${ }^{3,4}$ D $\cdot$ Lars Pastewka ${ }^{3,4} \mathbb{D}$
}

Received: 30 January 2021 / Accepted: 5 May 2021 / Published online: 21 May 2021

(c) The Author(s) 2021

\begin{abstract}
Understanding the distribution of interfacial separations between contacting rough surfaces is integral for providing quantitative estimates for adhesive forces between them. Assuming non-adhesive, frictionless contact of self-affine surfaces, we derive the distribution of separations between surfaces near the contact edge. The distribution exhibits a power-law divergence for small gaps, and we use numerical simulations with fine resolution to confirm the scaling. The characteristic length scale over which the power-law regime persists is given by the product of the rms surface slope and the mean diameter of contacting regions. We show that these results remain valid for weakly adhesive contacts and connect these observations to recent theories for adhesion between rough surfaces.
\end{abstract}

Keywords Surface roughness · Contact mechanics · Adhesion

\section{Introduction}

Contact between nominally flat, rough surfaces has been the subject of study for countless experimental, analytical, and numerical investigations over the past century [1-31]. A universal theme found in most cases is that contact is limited to the peaks or asperities of the rough topography and the real area of contact $A_{\text {rep }}$ is much less than the apparent projected area $A_{0}$. Substantial progress has been made in determining the relationship between $A_{\text {rep }}$ and the applied normal force $F$ in non-adhesive, frictionless systems

Lars Pastewka

lars.pastewka@imtek.uni-freiburg.de

Joseph M. Monti

jmonti3@jhu.edu

1 Department of Physics and Astronomy, Johns Hopkins University, 3400 N. Charles St., Baltimore, MD 21218, USA

2 Present Address: Sandia National Laboratories, Albuquerque, USA

3 Department of Microsystems Engineering, University of Freiburg, Georges-Köhler-Allee 103, 79110 Freiburg, Germany

4 Cluster of Excellence livMatS, Freiburg Center for Interactive Materials and Bioinspired Technologies, University of Freiburg, Georges-Köhler-Allee 105, 79110 Freiburg, Germany assuming elastic response. In such cases, the proportionality $A_{\text {rep }}=\kappa_{\text {rep }} F / h_{0}^{\prime} E^{*}$ is found when $A_{\text {rep }} \lesssim 0.1 A_{0}$. Here, $h_{0}^{\prime}$ is the root mean square (rms) slope of the rough topography, $E^{*}$ is the elastic contact modulus, and the dimensionless constant $\kappa_{\text {rep }} \approx 2[2,6,7]$. This result implies a load-independent mean compressive stress, $\sigma_{\text {rep }}=h_{0}^{\prime} E^{*} / \kappa_{\text {rep }}$ in contacting regions. Since flattening a region with slope $h_{0}^{\prime}$ introduces a strain of order $h_{0}^{\prime}$ into the surface, $\sigma_{\text {rep }}$ reflects the stress required to flatten the rough topography.

Recently, interest has turned to systems including attractive interactions that lead to macroscopic adhesion. Two opposite limits exist in the classical literature on the adhesion of smooth spheres: in the Derjaguin-Muller-Toporov (DMT) [32] limit, weak attractive forces between solids do not alter the geometry of contact, but do reduce the global mean pressure; in the opposite limit, known as the Johnson-Kendall-Roberts (JKR) [33] limit, strong attractive forces significantly change the structure of the contact edge and can lead to contact hysteresis. For the contact of spheres, the Tabor and Maugis parameters [34] describe the continuous transition between the two limits in terms of the relative strengths of adhesive and elastic parameters.

Working in a DMT-like limit, Pastewka and Robbins developed a theory to predict the onset of stickiness in contacts of self-affine rough surfaces [23] that was later independently confirmed by Müser [35]. They split the total normal force as $F=F_{\text {rep }}+F_{\text {att }}$ into the sum of a repulsive contribution 
$F_{\text {rep }}>0$ and an attractive contribution $F_{\text {att }}<0$. Repulsive and attractive contributions originate from repulsive surface patches of total area $A_{\text {rep }}$ and attractive surface patches of area $A_{\text {att }}$ (see Fig. 1). The total force is then given by

$F=\sigma_{\text {rep }} A_{\text {rep }}-\sigma_{\text {att }} A_{\text {att }}$,

where $\sigma_{\text {att }}$ is the mean stress in the attractive patches that is roughly constant and of order $\sigma_{\text {att }}=w / \Delta r$, where $w$ is the work of adhesion and $\Delta r$ the range of the attractive interaction.

Like in the purely non-adhesive limit, the geometry of contact is fractal in the DMT-like limit with proportionality between $A_{\text {rep }}$ and the contact perimeter $P_{\text {rep }}$ given by

$P_{\text {rep }}=\pi A_{\text {rep }} / d_{\text {rep }}$,

where the mean contact diameter $d_{\text {rep }}$ is approximately constant $[7,23,36]$. Note that Eq. (2) holds generally for any geometric object, but $d_{\text {rep }}$ varies with $A_{\text {rep }}$ in most cases. Short-ranged attractive interactions generate narrow bands of approximately constant width $d_{\text {att }}$ located around contact regions (see Fig. 1). If $d_{\text {att }}$ is small, then the total area contributing to attractive forces $A_{\text {att }}=P_{\text {rep }} d_{\text {att }}$, which can be related to $A_{\text {rep }}$ via mutual proportionality with $P_{\text {rep }}$ given



Fig. 1 a Contact map for a self-affine surface with $H=0.8$ and $\lambda_{\min }=16 a_{0} . A_{\text {rep }}$ is the sum over all pixels shown in black, and the contact perimeter $P_{\text {rep }}$ is marked in red. b Schematic of a contact region created by a contacting asperity, showing the mean contact diameter $d_{\text {rep }}$. The gap $\Delta(x)$ between surfaces grows as the lateral distance $x^{3 / 2}$ (Color figure online) by Eq. (2). This means, that for non-sticky interfaces, the (repulsive) contact area is given by the expression

$A_{\text {rep }}=\kappa \frac{F}{h_{0}^{\prime} E^{*}}$

with an effective $1 / \kappa=1 / \kappa_{\text {rep }}-1 / \kappa_{\text {att }}$. The adhesive interaction hence increases the effective value of the dimensionless constant $\kappa$. A macroscopic force is required to separate the two surface when $\left|F_{\text {att }}\right| \approx F_{\text {rep }}$ or equivalently $\kappa_{\text {att }} \approx \kappa_{\text {rep }}$. Interfaces that require a macroscopic force for separation are called "sticky".

This theory depends sensitively upon the distribution of interfacial separations or gaps, that is assumed to be unaltered from the non-adhesive scenario. Previous work has primarily focused on the behavior of the mean gap $\bar{g}$, which is commonly found to be exponentially related to the normal load in non-adhesive contact as $F \propto \exp \left(-\bar{g} / \gamma h_{0}\right)$, where $h_{0}$ is the rms surface height and $\gamma$ is a dimensionless constant of order unity [8, 9, 12-17, 21, 24, 29]. Almqvist et al. [16], the contact mechanics challenge [29] and Wang and Müser [37] have reported distributions of interfacial separations, but these works have either not focused on the behavior at small gaps that is important for understanding short-ranged adhesion or indirectly reported it through analysis of percolation in Reynolds flow.

In this paper, we derive the distribution of interfacial separations in the vicinity of contacting regions and show numerically that our expression holds even in the weakly adhesive limit. This distribution can be used to compute the total attractive contribution to the force and hence the force-area relationship for weakly adhesive interfaces.

\section{Simulation Methods}

In our simulations, we invoke the standard mapping that allows the contact of two rough, elastic solids to be treated as contact between an initially flat, elastic solid and a rough, rigid surface [38]. If the elastic properties of the two original surfaces are encoded by the Young's moduli $E_{1}$ and $E_{2}$ and Poisson's ratios $v_{1}$ and $v_{2}$, the combined elastic response is given by the elastic contact modulus

$\frac{1}{E^{*}}=\frac{1-v_{1}^{2}}{E_{1}}+\frac{1-v_{2}^{2}}{E_{2}}$.

The roughness profile of each periodic, $L \times L$ surface with nominal area $A_{0}=L^{2}$ is described by a self-affine fractal between an upper cutoff length scale $\lambda_{\max }$ and a lower cutoff $\lambda_{\min }$, where length scales are given in terms of the pixel size $a_{0}$. This means that the power spectral density (PSD) 
$C(\mathbf{q})$ of the isotropic, self-affine roughness depends only on wavevector magnitude $q=|\mathbf{q}|$ and satisfies

$C(q)= \begin{cases}C_{0} & \text { if } q \leq q_{1} \\ C_{0}\left(\frac{q}{q_{1}}\right)^{-2(1+H)} & \text { if } q_{1}<q \leq q_{2}, \\ 0 & \text { if } q>q_{2}\end{cases}$

where $q_{1}=2 \pi / \lambda_{\max }$ and $q_{2}=2 \pi / \lambda_{\min }$ are the wavevector magnitudes corresponding to the roughness cutoffs and $H$ is the Hurst exponent $(0<H<1)$ that determines correlations in the roughness. The resolution of the calculations is given by $\lambda_{\min } / a_{0}$, while the ratio $L / \lambda_{\max }$ controls the surface "representativity" [18]. The distribution of heights $P(h)$ for a Gaussian self-affine rough surface with mean height $\bar{h}$ and $L / \lambda_{\max } \gg 1$ has (by construction) Gaussian form. We use $\lambda_{\min } \geq 32 a_{0}$ for the results presented here to ensure that the contact edge is sufficiently resolved. We use a Fourierfiltering algorithm to create such self-affine surfaces for our calculations [36, 39].

Performing the mapping described above results in a rough surface that is the incoherent sum of the rough profiles of the original surfaces. For the combination of two profiles that have identical statistical properties, the commonly utilized statistical measures of roughness - the rms height $h_{0}=\sqrt{\left\langle h^{2}\right\rangle}$, rms slope $h_{0}^{\prime}=\sqrt{\left\langle|\nabla h|^{2}\right\rangle}$, and rms curvature $h_{0}^{\prime \prime}=\sqrt{\left\langle\left|\nabla^{2} h\right|^{2}\right\rangle} / 2$ - of the combined surface each increase by a factor of $\sqrt{2}$. We therefore here work in the limit of a rigid rough surface contacting a flat deformable elastic halfspace and all statistical properties are to be interpreted for a combined surface. Note that $\langle\cdot\rangle$ denotes the spatial average over the domain where the topography function $h(x, y)$ is defined.

We use a static boundary element method to compute the linear elastic deformation induced by normal contact on an isotropic half-space (Refs. [40-42]). In order for linear elasticity to be a good approximation, the surface slope must be small. We use $h_{0}^{\prime}=0.1$ to ensure that this approximation is justified, but note that real surfaces may have values $h_{0}^{\prime} \approx 1$ or larger $[31,43,44]$.

Our simulations assume frictionless contact with a noninterpenetration constraint. Pixels on the surface of the elastic solid are considered to be in contact when they bear a compressive pressure; each contacting pixel contributes an area $a_{0}^{2}$ to the total contact area. For non-adhesive calculations, we solve the interpenetration constraint using a constrained conjugate-gradient optimizer [45]. For adhesive calculations, we assume an interaction energy of

$v(g)=-w \exp (-g / \rho)$

that depends on the gap $g$, where $\rho$ is the interaction range. (Note that the range defined in Ref. [23] is $\Delta r \approx 1.36 \rho$ for this potential.) The overall adhesive energy is then given by
$E_{\mathrm{att}}=\int d x d y v(g(x, y))$

where $g(x, y)$ is the local gap at position $x, y$. This attractive interaction is minimized with an interpenetration constraint realized through the constrained non-linear conjugate-gradient algorithm of Ref. [46]. Similar boundary element methods have been used extensively to study rough contacts [11, $18-23,25-28,30]$. The attractive interaction is identical to the one employed in the contact mechanics challenge [29].

\section{Theory}

The distribution of interfacial separations $g$ between a rough surface and an undeformed elastic solid with surface at $h=0$ is equal to the (Gaussian) distribution of heights,

$p(g)=\frac{1}{\sqrt{2 \pi} h_{0}} \exp \left[-\frac{(g-\bar{g})^{2}}{2 h_{0}^{2}}\right]$ for $g \geq 0$,

where $\bar{g}$ is the initial mean surface height. The width of the distribution is the same as for the rough surface itself and scales with the long wavelength cutoff, $h_{0} \sim \lambda_{\text {max }}^{H}$.

When the solids are pushed together under load, the elastic solid deforms and the mean interfacial separation $\bar{g}$ shrinks. We now write the gap distribution as the additive decomposition

$p(g)=p_{c}(g)+p_{n}(g)+p_{f}(g)$,

where $p_{c}(g)$ contains the distribution of the gaps $g$ within the contacting area, $p_{n}(g)$ the contribution from near the contact edge, and $p_{f}(g)$ the contribution from farther distances from the contact edge. Since $p(g)$ is normalized, the probability of contact for a given contact fraction $c=A_{\text {rep }} / A_{0}$ is

$p_{c}(g)=c \delta(g)$,

where $\delta$ is the Dirac $\delta$-function.

The next contribution $p_{n}(g)$ comes from small separations near the contact edge. As shown in numerical calculations in Refs. [7, 23], the contact edge has a total perimeter $P_{\text {rep }}=\pi A_{\text {rep }} / d_{\text {rep }}$ with a constant $d_{\text {rep }}$. (This means that both area and contact edge are fractal objects—see Fig. 1a—and suggests that their fractal dimensions are identical.) We can write this contribution as

$p_{n}(g)=\frac{P_{\text {rep }}}{A_{0}} \int_{0}^{\xi} d x \delta(g-\Delta(x))$

where $\Delta(x)$ describes how the mean gap between the two contacting surfaces varies as a function of distance $x$ from the contact edge (see Fig. 1b). Note that we take the integral in Eq. (11) out to a characteristic length $\xi$ that is close 
enough to the contact edge such that the number of points contributing to the gap distribution is still proportional to $P_{\text {rep. }}$

The arguments that lead to Eq. (11) rely on the geometry of the contact patches formed between contacting self-affine surfaces. Refs. [7, 23] showed that in this case $P_{\text {rep }} \propto A_{\text {rep }}$, compared to $P_{\text {rep }} \propto A_{\text {rep }}^{1 / 2}$ as expected for simple contact shapes like circles. The larger perimeter scaling exponent arises because fractal contact regions are not compact, and a significant perimeter contribution comes from regions inside the convex hull enclosing individual patches. As patches become larger, they simply contain more non-contacting regions. The presence of non-contacting regions makes the contact geometry appear locally rectangular. The deformation cross-section for each line segment drawn through a single continuous contact diameter looks like that of a cylindrical indenter, rather than a spherical one. Within Euclidean geometry, a rectangle with constant thickness along its minor axis has the property $P_{\text {rep }} \propto A_{\text {rep }}$, if the rectangle is thin enough such that the contributions to $P_{\text {rep }}$ from the shorter sides can be neglected. The characteristic width of the contacting rectangle, and hence the contact diameter for the cylinder, is $d_{\text {rep }}$.

Working with this analogy, we now derive an analytic prediction for the distribution of gaps produced by nonadhesive contact of smooth surfaces. Assuming a non-adhesive cylindrical contact $[47,48]$, we have

$\Delta(x)=\frac{4 h_{0}^{\prime} d_{\text {rep }}}{3}\left(\frac{x}{d_{\text {rep }}}\right)^{3 / 2}=g_{0}\left(\frac{x}{d_{\text {rep }}}\right)^{3 / 2}$,

where $h_{0}^{\prime}$ is the local slope of the cylinder at the contact edge. It can be shown generally for non-adhesive contact that the local separation $\Delta(x) \propto x^{3 / 2}$ for small lateral distances $x$ from the contact edge [38]. The characteristic scale for the interfacial separation at the contact edge is $g_{0}=4 h_{0}^{\prime} d_{\text {rep }} / 3$, the prefactor in Eq. (12). Inserting Eqs. (12) into (11) yields

$p_{n}(g)=\frac{2 P_{\text {rep }} d_{\text {rep }}}{3 A_{0} g_{0}}\left(\frac{g_{0}}{g}\right)^{1 / 3}=\frac{2 \pi c}{3 g_{0}}\left(\frac{g_{0}}{g}\right)^{1 / 3}$,

where Eq. (2) was used for the right-hand equality. This is our prediction for the gap distribution near the contact edge. For small $g$, the distribution diverges as $g^{-1 / 3}$.

We note that $\int_{0}^{\infty} d g p(g)=1$, but taking the integral out to infinity for the contribution to $p(g)$ given by Eq. (13) diverges. The contribution $p_{n}(g)$ to the gap distribution can therefore only be valid up to $g \sim g_{0}$, the characteristic gap that is reached a distance $d_{\text {rep }}$ from the contact edge. The "far" contribution $p_{f}(g)$ looks like the undeformed distribution of heights given by Eq. (8) (see also Refs. [16, 29]).

Almqvist et al. [16] have observed a divergence of the gap distribution for small $g$ but have not quantified the exponent.
Pastewka and Robbins [23] have used this divergence for their theory of "stickiness" but have not provided extensive numerical evidence for its validity. We now supplement these observations with additional high-resolution numerical data.

\section{Results}

We performed simulations with sufficiently fine resolution ( $\lambda_{\min }=32 a_{0}$ and $128 a_{0}$ ) to test Eq. (13). Figure 2 shows distributions of interfacial separations for non-contacting grid points. The distributions were normalized to the respective non-contacting fractional area, $1-c$, and divided by the prefactor of Eq. (13) to collapse all data points onto a single $\left(g / g_{0}\right)^{-1 / 3}$ power-law. We used the numerically measured value of $d_{\text {rep }}$ (see Ref. [23] on details of how to compute $d_{\text {rep }}$ ) for the data collapse. The power-law regime emerges for both $H=0.3$ (Fig. 2a) and $H=0.8$ (Fig. 2b).

The data only collapse over a limited range of gaps. The divergence at small gap predicted by Eq. (13) is always cut off at a minimum length scale, below which $p(g)$ is uniformly distributed. In our simulations, the cutoff scale is $\sim 10^{-3}-10^{-2} a_{0}$; the threshold for saturation of $p(g)$ at small $g / g_{0}$ is inversely proportional to the resolution $a_{0} / \lambda_{\min }$. For $H=0.3$ (Fig. 2a), contributions to $p(g)$ from near-contact and far-from-contact overlap (i.e., $p_{n}(g)$ and $p_{f}(g)$ have similar magnitude) for $\lambda_{\min }=32 a_{0}$ (solid symbols). The power-law regime only clearly emerges over $1-2$ decades for $H=0.3$ if the resolution of the calculation is improved by increasing $\lambda_{\min }$, as is shown for $\lambda_{\min }=128 a_{0}$ (open symbols). For $H=0.8$, on the other hand, the power-law regime extends over a much larger range of gaps even for our "coarse" calculations with $\lambda_{\min }=32 a_{0}$. As for $H=0.3$, increasing the short-wavelength cutoff to $\lambda_{\text {min }}=128 a_{0}$ extends the power-law to smaller gaps.

One can integrate $p_{n}(g)$ to calculate the cumulative fractional area $c_{n}\left(g_{c}\right)$ in the near-contacting region closer than a cutoff gap $g_{c}$. We find

$c_{n}\left(g_{c}\right)=\int_{0}^{g_{c}} d g p_{n}(g)=\pi c\left(\frac{g_{c}}{g_{0}}\right)^{2 / 3}$,

which for $g_{c}=g_{0}$ yields $c_{n} / c=\pi$. This means the area in the near-contact region is $\sim 3$ times the area in the contacting regions. The area in the near-contact region is equal to the contact area when $g_{c} \approx 0.18 g_{0}$. The breakdown of the power-law region at small $g$ occurs at $g / g_{0} \approx 10^{-3}$ or smaller (for $H=0.8$ ), meaning that the area within the rolloff region at small $g$ is at most about $3 \%$ of the contact area.

For large gaps the power-law regime is cut off by a Gaussian gap distribution that reflects the distribution of undeformed or weakly deformed parts of the surface (see also 

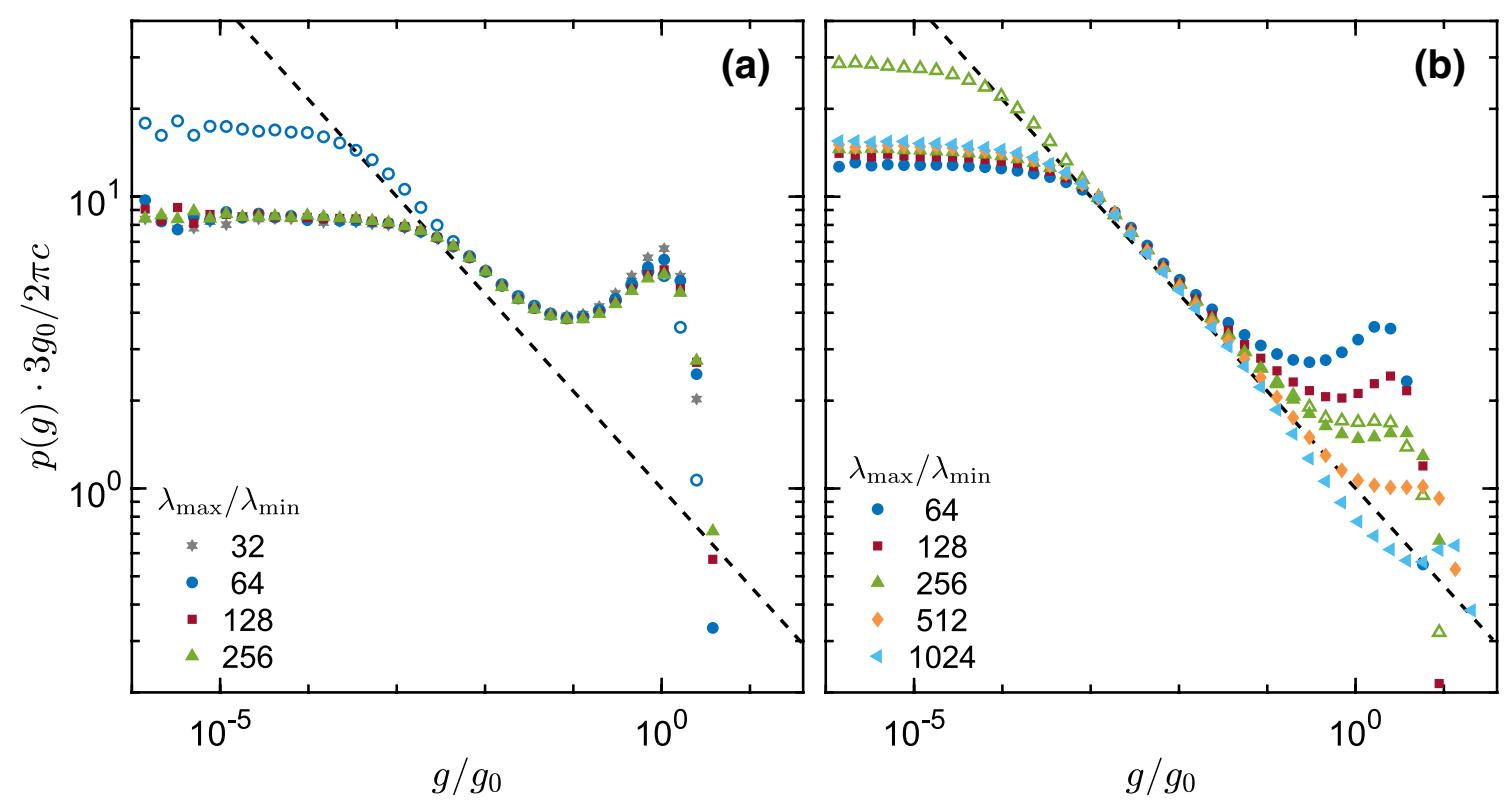

Fig. 2 The probability distribution of interfacial separations for $H=0.3$ (a) and $H=0.8$ (b) normalized to $1-c$ and divided by the prefactor in Eq. (13) for the ratios $\lambda_{\max } / \lambda_{\min }$ indicated in the legend, with $\lambda_{\min }=32 a_{0}$ (solid symbols) and $\lambda_{\min }=128 a_{0}$ (open sym-

Eq. (8)). For both $H=0.3$ and $H=0.8$, the uptick in $p(g)$ in the range $g / g_{0} \sim 1-10$ is the peak of this Gaussian height distribution. Since $h_{0} \propto\left(\lambda_{\max } / \lambda_{\min }\right)^{H}$, the power-law regime extends much further for $H=0.8$ (Fig. 2b) than for $H=0.3$ (Fig. 2a). Increasing $\lambda_{\max } / \lambda_{\min }$ shifts the Gaussian peak out to larger $g / g_{0}$ (most prominently for $H>0.5$ ), and extends the range over which $p(g) \approx p_{n}(g)$. However, for the largest $\lambda_{\max } / \lambda_{\text {min }}$ we studied, $p(g)<p_{n}(g)$ for $0.1 \lesssim g / g_{0} \lesssim 1$. This suggests that the cutoff of the integral in Eq. (11) may be up to an order of magnitude smaller than $g_{0}$, about equal to the point where the near-contact area matches the contact area. Still larger calculations may be required to verify this result.

The crucial question for adhesive theories is how applicable our results are for gap distributions in the presence of attractive interactions. We quantify the strength of the attractive interaction by the value of $1 / \kappa_{\text {att }}$ (see Ref. [23] and discussion below), since interfaces become sticky for $1 / \kappa_{\text {att }} \gtrsim 1 / 2$ [23]. For the data collapse, we use the values of $d_{\text {rep }}$ measured in the non-adhesive calculations.

Figure 3 shows the gap distributions for adhesive calculations as $w$ increases (with constant $\rho$ ), using surfaces with $H=0.8$ and $\lambda_{\min }=128 a_{0}$. To facilitate the comparison between non-adhesive and adhesive calculations, the adhesive simulations are conducted with the constraint that the mean gap is identical to the non-adhesive simulation $\left(1 / \kappa_{\text {att }}=0\right)$. This choice of constraint means that the contact areas are not equal, particularly for sticky surfaces. For non-sticky surfaces (up to $1 / \kappa_{\text {att }} \approx 0.2$ ), the gap distribution bols, color matches $\lambda_{\max } / \lambda_{\min }$ in the legend). Here, $A / A_{0} \approx 0.03$ for $\lambda_{\text {min }}=32 a_{0}$ and $A / A_{0} \approx 0.04$ for $\lambda_{\text {min }}=128 a_{0}$. The power-law $p_{n}(g)$ from Eq. (13) is shown as a dashed black line

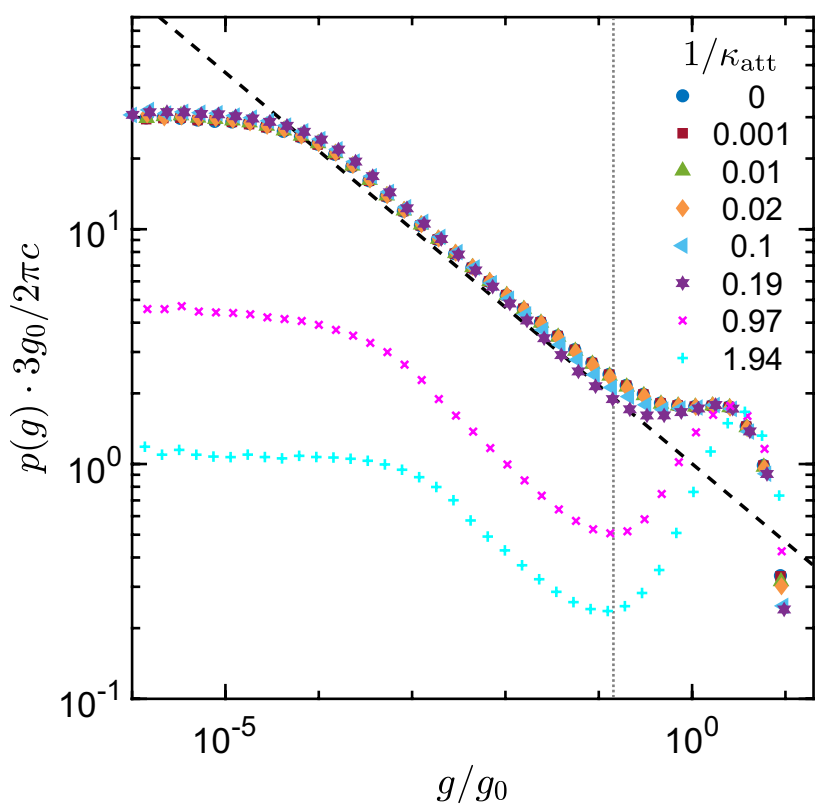

Fig. 3 Comparison of the interfacial probability distributions for adhesive contact with increasing $w$, for $H=0.8, \lambda_{\min }=128 a_{0}$, $\lambda_{\max } / \lambda_{\min }=256$, and $L / \lambda_{\max }=2$. The corresponding non-adhesive contact distribution from Fig. $2 b$ is replotted $\left(1 / \kappa_{\text {att }}=0\right.$, $A_{\text {rep }} / A_{0} \approx 0.04$ ), and the adhesive distributions are normalized by $g_{0}$ and $c$ obtained from the non-adhesive result. The power-law $p_{n}(g)$ from Eq. (13) is shown as a dashed black line. The dotted gray line corresponds to $g / g_{0}=\rho / g_{0}$ with $\rho=4 a_{0}$. 
follows the predicted power-law over the same range as the non-adhesive result. At $1 / \kappa_{\text {att }} \approx 0.2\left(A_{\text {rep }} / A_{0} \approx 0.06\right)$ there is a slight deviation toward smaller gaps. The sticky cases with $1 / \kappa_{\text {att }} \approx 1$ and $1 / \kappa_{\text {att }} \approx 2\left(A_{\text {rep }} / A_{0} \approx 0.12\right.$ and 0.16 , respectively) clearly deviate from our prediction for the divergence. These sticky interfaces appear to still exhibit a regime where $p(g) \propto g^{-1 / 3}$, but the range of the power-law regime becomes narrower as $1 / \kappa_{\text {att }}$ increases. This means that the prefactor from Eq. (13) no longer captures the intensity of the divergence and much of the non-contacting area is pushed out toward larger gaps. This is also reflected by the increase of the peak at larger gaps. The characteristic gap below which the distribution rolls-off and becomes constant appears to increase slightly in the sticky limit.

\section{Discussion and Conclusions}

The behavior of the near-contact interfacial separation is an important consideration in the context of adhesive contact because it determines the attractive contribution to the force,

$\frac{F_{\text {att }}}{A_{0}}=\int_{0}^{\infty} d g p(g) \frac{d v}{d g}$.

Assuming that the contribution from $p_{f}(g)$ is negligible, i.e., that our potential is sufficiently short-ranged, the attractive pressure per unit contact area is then given by

$\frac{F_{\text {att }}}{A_{\text {rep }}}=\frac{2 \pi}{3} \int_{0}^{\infty} \frac{d g}{g_{0}}\left(\frac{g_{0}}{g}\right)^{1 / 3} \frac{d v}{d g}=-\frac{2 \pi}{3} \frac{w}{\left(g_{0}^{2} \rho\right)^{1 / 3}}$,

where we have used the interaction law $v(g)$ from Eq. (6). Note that Eq. (16) defines the value of the dimensionless constant $1 / \kappa_{\text {att }}=F_{\text {att }} / h_{0}^{\prime} E^{*} A_{\text {rep }}$, which we used to quantify the strength of adhesion in Fig. 3 and which can be used to determine the effective range of adhesion $\Delta r$ (see Ref. [23]).

This expression can also be used to quantify what "short-ranged" adhesion means for rough surfaces and, therefore, to determine the limits of the theories of Pastewka and Robbins [23] and Müser [35]. Figure 4 shows the normalized integrand of Eq. (16) as a function of the normalized gap $g / g_{0}$. The integrand depends on the range of the interaction potential $\rho$. For $\rho \approx 0.1 g_{0}$ and below, the main contribution to the integrand and thereby the attractive force comes from gaps with $g / g_{0}<0.1$ where the power-law holds. The roll-off region at small gap contributes negligibly to this integral. This means that the adhesion range is short enough if $\rho \lesssim 0.1 g_{0}$. The calculations presented here were carried out with $\rho=4 a_{0}$ (as is typical for attractive interactions, e.g., Refs. $[49,50])$ and our adhesive calculations have $g_{0}>25 a_{0}$, which means the range is sufficiently small. Interactions with a larger

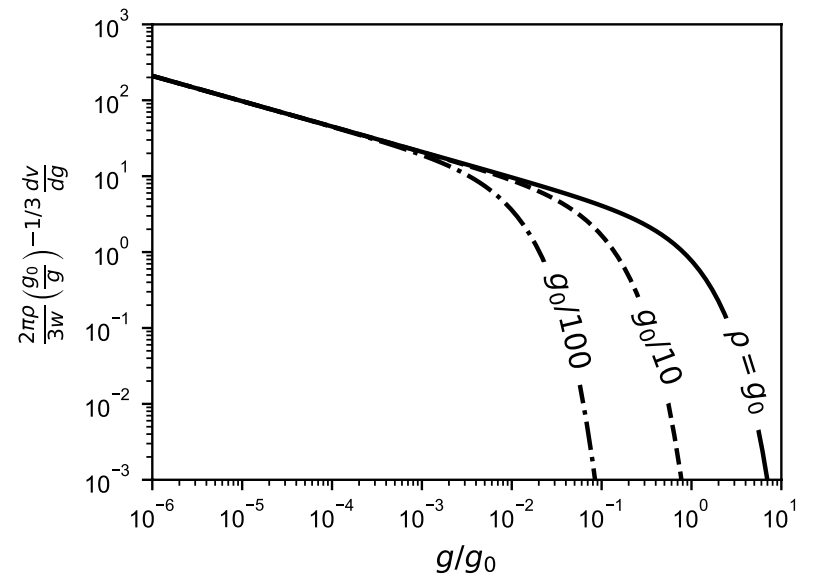

Fig. 4 Integrand of Eq. (16), i.e., non-dimensionalized contribution to the overall attractive force as a function of gap $g / g_{0}$ for different ranges $\rho$ of the adhesive interaction

range (e.g., electrostatic interactions) interact with the full topography of the surface and require corrections to the expression for $F_{\text {att }}$. The same is true for extremely smooth surfaces with small values of $g_{0}$. This defines bounds for theory outlined in Refs. [23, 35].

Besides leaving the gap distribution unmodified, the DMT-like limit also implies that the repulsive area $A_{\text {rep }}$ equals the typical definition of the contact area, namely, vanishing gaps $g=0$. The latter definition makes sense in continuum theories (like the present work), but not for models that consider the full intermolecular interaction, which exhibits soft repulsion (e.g., Ref. [23]), or for those that include thermal fluctuations (e.g., Refs. [51, 52]). Like in the JKR model [33], these definitions of contact no longer agree for sticky interfaces. In addition, JKR-like contacts separate as $x^{1 / 2}$ and not $x^{3 / 2}$ near the contact edge [53], leading to a gap distribution $p_{n}(g) \propto g$ that is clearly distinct from what we observe in our calculations. Furthermore, in the sticky limit, the contact geometry changes substantially and is not expected to give rise to the simple proportional contact area-perimeter relationship used in our calculations. This is especially true for soft solids, which can deform to fill in interior non-contacting regions without large elastic energy penalties, thereby increasing the contact area at the expense of the overall contact perimeter.

Nevertheless, the present calculations are a powerful demonstration of the universal emergence of the $g^{-1 / 3}$ divergence in the distribution of gaps between elastically stiff rough surfaces. Since this behavior is a direct consequence of the fractal character of the contacting interfaces as manifested in the proportionality between perimeter and contact area, our results are another indirect demonstration of this aspect of the contact geometry. The distribution is unaltered by weak adhesive interactions, giving additional support for 
the DMT-like approximation that underlies the adhesive theories of Pastewka and Robbins [23] and Müser [35].

Acknowledgements This work has emerged from numerous enlightening interactions with Mark Robbins over the last decade. All of us were inspired by him and JMM and LP will forever be thankful for having been given the opportunity to closely work with Mark. We also thank Martin Müser for useful discussions and Sindhu Singh for implementing the optimization algorithm of Ref. [46].

Author Contributions JMM and LP devised the study. JMM and AS carried out and analyzed contact mechanics calculations. JMM wrote the first manuscript draft. All authors contributed to editing and finalizing the manuscript.

Funding Open Access funding enabled and organized by Projekt DEAL. We thank the DAAD for support for a short visit of JMM to Freiburg and the US National Science Foundation (Grant DMR1411144 and DMR-1929467), the European Commission (Marie-Curie IOF-272619), and the Deutsche Forschungsgemeinschaft (Grants PA 2023/2 and EXC 2193/1-390951807) for funding. Computations were carried out on the Johns Hopkins University Homewood High Performance Cluster, the BlueCrab cluster at the Maryland Advanced Research Computing Center, and NEMO at the University of Freiburg (DFG Grant INST 39/963-1 FUGG).

Code Availability We used the CONTACT.ENGINEERING ecosystem for all calculations. The code is available at https://github.com/ContactEng ineering under the terms of the MIT license.

Data Availibility Data are available from the authors upon request.

\section{Declarations}

Conflict of interest The authors declare no competing interests.

Open Access This article is licensed under a Creative Commons Attribution 4.0 International License, which permits use, sharing, adaptation, distribution and reproduction in any medium or format, as long as you give appropriate credit to the original author(s) and the source, provide a link to the Creative Commons licence, and indicate if changes were made. The images or other third party material in this article are included in the article's Creative Commons licence, unless indicated otherwise in a credit line to the material. If material is not included in the article's Creative Commons licence and your intended use is not permitted by statutory regulation or exceeds the permitted use, you will need to obtain permission directly from the copyright holder. To view a copy of this licence, visit http://creativecommons.org/licenses/by/4.0/.

\section{References}

1. Greenwood, J.A., Williamson, J.B.P.: Contact of nominally flat surfaces. Proc. R. Soc. Lond. A 295(1442), 300-319 (1966)

2. Bush, A.W., Gibson, R.D., Thomas, T.R.: The elastic contact of a rough surface. Wear 35(1), 87-111 (1975)

3. Dieterich, J.H., Kilgore, B.D.: Direct observation of frictional contacts: new insights for state-dependent properties. Pure Appl. Geophys. 143(1-3), 283-302 (1994)

4. Dieterich, J.H., Kilgore, B.D.: Imaging surface contacts: power law contact distributions and contact stresses in quartz, calcite, glass and acrylic plastic. Tectonophysics 256(1-4), 219-239 (1996)

5. Persson, B.N.J.: Theory of rubber friction and contact mechanics. J. Chem. Phys. 115(8), 3840-3861 (2001)

6. Persson, B.N.J.: Elastoplastic contact between randomly rough surfaces. Phys. Rev. Lett. 87(11), 116101-1-116101-4 (2001)

7. Hyun, S., Pel, L., Molinari, J.-F., Robbins, M.O.: Finite-element analysis of contact between elastic self-affine surfaces. Phys. Rev. E 70(2), 1-12 (2004)

8. Pei, L., Hyun, S., Molinari, J.-F., Robbins, M.O.: Finite element modeling of elasto-plastic contact between rough surfaces. J. Mech. Phys. Solids 53(11), 2385-2409 (2005)

9. Benz, M., Rosenberg, K.J., Kramer, E.J., Israelachvili, J.N.: The deformation and adhesion of randomly rough and patterned surfaces. J. Phys. Chem. B 110(24), 11884-11893 (2006)

10. Hyun, S., Robbins, M.O.: Elastic contact between rough surfaces: effect of roughness at large and small wavelengths. Tribol. Int. 40(10-12), 1413-1422 (2007)

11. Campañá, C., Müser, M.H.: Contact mechanics of real vs. randomly rough surfaces: a Green's function molecular dynamics study. Europhys. Lett. 77(3), 38005 (2007)

12. Persson, B.N.J.: Relation between interfacial separation and load: a general theory of contact mechanics. Phys. Rev. Lett. 99(12), 125502 (2007)

13. Yang, C., Persson, B.N.J.: Molecular dynamics study of contact mechanics: contact area and interfacial separation from small to full contact. Phys. Rev. Lett. 100(2), 024303 (2008)

14. Yang, C., Persson, B.N.J.: Contact mechanics: contact area and interfacial separation from small contact to full contact. J. Phys. 20(21), 215214 (2008)

15. Lorenz, B., Persson, B.N.J.: Interfacial separation between elastic solids with randomly rough surfaces: comparison of experiment with theory. J. Phys. 21(1), 015003 (2009)

16. Almqvist, A., Campañá, C., Prodanov, N., Persson, B.N.J.: Interfacial separation between elastic solids with randomly rough surfaces: comparison between theory and numerical techniques. J. Mech. Phys. Solids 59(11), 2355-2369 (2011)

17. Akarapu, S., Sharp, T., Robbins, M.O.: Stiffness of contacts between rough surfaces. Phys. Rev. Lett. 106(20), 204301 (2011)

18. Yastrebov, V.A., Anciaux, G., Molinari, J.-F.: Contact between representative rough surfaces. Phys. Rev. E 86(3), 035601 (2012)

19. Pohrt, R., Popov, V.L.: Normal contact stiffness of elastic solids with fractal rough surfaces. Phys. Rev. Lett. 108(10), 104301 (2012)

20. Prodanov, N., Dapp, W.B., Müser, M.H.: On the contact area and mean gap of rough, elastic contacts: dimensional analysis, numerical corrections, and reference data. Tribol. Lett. 53(2), 433-448 (2013)

21. Pastewka, L., Prodanov, N., Lorenz, B., Müser, M.H., Robbins, M.O., Persson, B.N.J.: Finite-size scaling in the interfacial stiffness of rough elastic contacts. Phys. Rev. E 87(6), 062809 (2013)

22. Yastrebov, V.A., Anciaux, G., Molinari, J.-F.: The contact of elastic regular wavy surfaces revisited. Tribol. Lett. 56(1), 171-183 (2014)

23. Pastewka, L., Robbins, M.O.: Contact between rough surfaces and a criterion for macroscopic adhesion. Proc. Natl. Acad. Sci. USA 111(9), 3298-3303 (2014)

24. Prodanov, N., Dapp, W.B., Müser, M.H.: On the contact area and mean gap of rough, elastic contacts: dimensional analysis, numerical corrections, and reference data. Tribol. Lett. 53(2), 433-448 (2014)

25. Yastrebov, V.A., Anciaux, G., Molinari, J.-F.: From infinitesimal to full contact between rough surfaces: evolution of the contact area. Int. J. Solids Struct. 52, 83-102 (2015) 
26. Pastewka, L., Robbins, M.O.: Contact area of rough spheres: large scale simulations and simple scaling laws. Appl. Phys. Lett. 108(22), 221601 (2016)

27. Yastrebov, V.A., Anciaux, G., Molinari, J.-F.: On the accurate computation of the true contact-area in mechanical contact of random rough surfaces. Tribol. Int. 114, 161-171 (2017)

28. Yastrebov, V.A., Anciaux, G., Molinari, J.-F.: The role of the roughness spectral breadth in elastic contact of rough surfaces. J. Mech. Phys. Solids 107, 469-493 (2017)

29. Müser, M.H., Dapp, W.B., Bugnicourt, R., Sainsot, P., Lesaffre, N., Lubrecht, T.A., Persson, B.N.J., Harris, K., Bennett, A., Schulze, K., Rohde, S., Ifju, P., Sawyer, W.G., Angelini, T., Ashtari Esfahani, H., Kadkhodaei, M., Akbarzadeh, S., Wu, J.J., Vorlaufer, G., Vernes, A., Solhjoo, S., Vakis, A.I., Jackson, R.L., $\mathrm{Xu}$, Y., Streator, J., Rostami, A., Dini, D., Medina, S., Carbone, G., Bottiglione, F., Afferrante, L., Monti, J., Pastewka, L., Robbins, M.O., Greenwood, J.A.: Meeting the contact-mechanics challenge. Tribol. Lett. 65(4), 1-18 (2017)

30. Weber, B., Suhina, T., Junge, T., Pastewka, L., Brouwer, A.M., Bonn, D.: Molecular probes reveal deviations from Amontons' law in multi-asperity frictional contacts. Nat. Commun. 9(1), 888 (2018)

31. Dalvi, S., Gujrati, A., Khanal, S.R., Pastewka, L., Dhinojwala, A., Jacobs, T.D.B.: Linking energy loss in soft adhesion to surface roughness. Proc. Natl. Acad. Sci. USA 116(51), 25484-25490 (2019)

32. Derjaguin, B.V., Muller, V.M., Toporov, Y.U.P.: Effect of contact deformation on the adhesion of particles. J. Colloid Interface Sci. 52(3), 105-108 (1975)

33. Johnson, K.L., Kendall, K., Roberts, A.D.: Surface energy and the contact of elastic solids. Proc. R. Soc. Lond. A 324, 301-313 (1971)

34. Maugis, D.: Adhesion of spheres: the JKR-DMT transition using a Dugdale model. J. Colloid Interface Sci. 150(1), 243-269 (1992)

35. Müser, M.H.: A dimensionless measure for adhesion and effects of the range of adhesion in contacts of nominally flat surfaces. Tribol. Int. 100, 41-47 (2016)

36. Ramisetti, S.B., Campañá, C., Anciaux, G., Molinari, J.-F., Müser, M.H., Robbins, M.O.: The autocorrelation function for island areas on self-affine surfaces. J. Phys. 23(21), 215004 (2011)

37. Wang, A., Müser, M.H.: Percolation and Reynolds flow in elastic contacts of isotropic and anisotropic, randomly rough surfaces. Tribol. Lett. 69(1), 1 (2020)

38. Johnson, K.L.: Contact Mechanics. Cambridge University Press, Cambridge (1985)

39. Jacobs, T.D.B., Junge, T., Pastewka, L.: Quantitative characterization of surface topography using spectral analysis. Surf. Topogr. 5(1), 013001 (2017)
40. Stanley, H.M., Kato, T.: An FFT-based method for rough surface contact. J. Tribol. 1(July), 2-6 (1997)

41. Campañá, C., Müser, M.H.: Practical Green's function approach to the simulation of elastic semi-infinite solids. Phys. Rev. B 74(7), 75420 (2006)

42. Pastewka, L., Sharp, T.A., Robbins, M.O.: Seamless elastic boundaries for atomistic calculations. Phys. Rev. B 86, 075459 (2012)

43. Gujrati, A., Khanal, S.R., Pastewka, L., Jacobs, T.D.B.: Combining TEM, AFM, and profilometry for quantitative topography characterization across all scales. ACS Appl. Mater. Interf. 10(34), 29169-29178 (2018)

44. Gujrati, A., Sanner, A., Khanal, S., Moldovan, N., Zeng, H., Pastewka, L., Jacobs, T.D.B.: Comprehensive topography characterization of polycrystalline diamond coatings. Surf. Topogr. 9(1), 014003 (2021)

45. Polonsky, I.A., Keer, L.M.: A numerical method for solving rough contact problems based on the multi-level multi-summation and conjugate gradient techniques. Wear 231(2), 206-219 (1999)

46. Bugnicourt, R., Sainsot, P., Dureisseix, D., Gauthier, C., Lubrecht, A.A.: FFT-based methods for solving a rough adhesive contact: description and convergence study. Tribol. Lett. 66(1), 29 (2018)

47. Baney, J.M., Hui, C.-Y.: A cohesive zone model for the adhesion of cylinders. J. Adhes. Sci. Technol. 11(3), 393-406 (1997)

48. Yang, F., Cheng, Y.-T.: Revisit of the two-dimensional indentation deformation of an elastic half-space. J. Mater. Res. 24(06), 1976-1982 (2009)

49. Grierson, D.S., Liu, J., Carpick, R.W., Turner, K.T.: Adhesion of nanoscale asperities with power-law profiles. J. Mech. Phys. Solids 61(2), 597-610 (2013)

50. Thimons, L.A., Gujrati, A., Sanner, A., Pastewka, L., Jacobs, T.D.B.: Hard material adhesion: which scales of roughness matter? Exp. Mech. (2021). https://doi.org/10.1007/s11340-021-00733-6

51. Cheng, S., Robbins, M.O.: Defining contact at the atomic scale. Tribol. Lett. 39(3), 329-348 (2010)

52. Zhou, Y., Wang, A., Müser, M.H.: How thermal fluctuations affect hard-wall repulsion and thereby hertzian contact mechanics. Front. Mech. Eng. 5, 67 (2019)

53. Tada, H., Paris, P.C., Irwin, G.R.: The Stress Analysis Of Cracks Handbook, 3rd edn. ASME Press, New York (2000)

Publisher's Note Springer Nature remains neutral with regard to jurisdictional claims in published maps and institutional affiliations. 